\section{REVISTA}

Revista Educación

ISSN: 0379-7082

ISSN: 2215-2644

revedu@gmail.com

Universidad de Costa Rica

Costa Rica

\title{
Estudio exploratorio sobre el conocimiento de los procesos de memoria en docentes
}

Muchiut, Álvaro Federico; Vaccaro, Paola; Zapata, Rocío Beatriz; Segovia, Ana Paula

Estudio exploratorio sobre el conocimiento de los procesos de memoria en docentes

Revista Educación, vol. 43, núm. 2, 2019

Universidad de Costa Rica, Costa Rica

Disponible en: http://www.redalyc.org/articulo.oa?id=44058158031

DOI: https://doi.org/10.15517/revedu.v43i2.32982

Esta obra está bajo una Licencia Creative Commons Atribución-NoComercial-SinDerivar 3.0 Internacional. 


\title{
Estudio exploratorio sobre el conocimiento de los procesos de memoria en docentes
}

\author{
Álvaro Federico Muchiut \\ Fundación Centro de Estudios Cognitivos - Instituto \\ Superior de Neuropsicología, Argentina \\ alvaro_muchutti@hotmail.com \\ (iD) http://orcid.org/0000-0002-2256-1226 \\ Paola Vaccaro \\ Fundación Centro de Estudios Cognitivos - Instituto \\ Superior de Neuropsicologia, Argentina \\ paolavaccaro1987@gmail.com \\ (D) http://orcid.org/0000-0003-1101-4603 \\ Rocio Beatriz Zapata \\ Fundación Centro de Estudios Cognitivos - Instituto \\ Superior de Neuropsicología, Argentina \\ rociob.zapata08@gmail.com \\ (iD http://orcid.org/0000-0002-1506-538X \\ Ana Paula Segovia \\ Fundación Centro de estudios Cognitivos - Instituto \\ Superior de Neuropsicología, Argentina \\ segovia.apaula@gmail.com \\ (iD http://orcid.org/0000-0002-3913-751X
}

An Exploratory Study on the Knowledge of Memory Processes Teachers

DOI: https://doi.org/10.15517/revedu.v43i2.32982
Redalyc: http://www.redalyc.org/articulo.oa?id=44058158031

DOI: https://doi.org/10.15517/revedu.v43i2.32982
Redalyc: http://www.redalyc.org/articulo.oa?id=44058158031 
Memory facilitates teaching and learning. This pre-experimental design study explores how knowledgeable teachers are about memory and consists of two stages that serve as baselines for future studies. A group of 196 teachers that taught all types of grade levels were asked to answer a two-part survey. The teachers were attendees at a Neuroeducation training session that was held in the city of Resistencia, Argentina in 2017. The data was analyzed on Excel spreadsheets. According to the results obtained, 65\% of the teachers stated having knowledge about the concept of memory and $15 \%$ reported having none. On the other hand, $72 \%$ said that they had minimal knowledge about memory as it relates to learning whereas $13 \%$ stated having none. The workshop proved to significantly increase the level of knowledge among the attendees regarding concept of memory (76\%), different types of memory (52\%) and how it relates to learning (55\%). It can be concluded that knowledge about how the student brain learns and, specifically, how the memory process is interrelated to learning, is essential for teacher training since it allows for better planning and more effective teaching.

KEYWORDs: Memory, learning processes, formal education, teacher.

\section{INTRODUCCIÓN}

En ocasiones, cuando se conversa con un/una docente o actor institucional sobre la práctica pedagógica (el trabajo en la escuela con el alumnado), surgen expresiones como No se acuerda de nada, El mes pasado lo vimos y ya no sabe, y otras que denotan preocupación en relación con los olvidos de lo ya enseñado en clases anteriores. Esto desembocó en la reflexión sobre qué es lo que pasa con la memoria dentro de las aulas de cada escuela.

Se decidió efectuar un sondeo sobre qué conoce el personal docente de los procesos de memoria y en cuál de ellos enfatiza en su práctica pedagógica; a modo de sentar las bases para investigaciones posteriores de mayor profundidad, donde pueda explorarse la relación memoria-aprendizaje.

Por lo tanto, el objetivo principal de este estudio fue explorar los conocimientos que el cuerpo docente tiene respecto del proceso de memoria y sus etapas. Planteándose como objetivos específicos:

a) cuáles son los conocimientos que posee el personal docente en relación con la memoria y qué conoce de las etapas del proceso de memoria;

b) conocer la relevancia que el cuerpo docente dice asignar a las etapas del proceso de memoria en relación con el aprendizaje; $y$

c) explorar en qué etapa del proceso de memoria reconoce cada docente enfatizar durante el desarrollo de las clases en la práctica pedagógica.

\section{JuSTIFICACIÓN}

Considerando que el aprendizaje es un proceso por el cual los organismos modifican su conducta para adaptarse a las condiciones cambiantes e impredecibles del medio que les rodea, y específicamente en el ser humano es una construcción y elaboración del sujeto que aprende, constituye una actividad reflexiva donde participa la conciencia (Davini, 2008). Ahora bien, lo que se aprende es retenido o almacenado en el cerebro y constituye lo que se denomina memoria. Por definición, no hay aprendizaje sin memoria ni memoria sin aprendizaje, aunque este último sea de naturaleza elemental. Aprendizaje y memoria son dos procesos estrechamente ligados y en cierto modo coincidentes, como las dos caras de una misma moneda (Morgado, 2005).

Por esta razón, resulta fundamental entender la relación que guardan estos dos procesos dentro del aula, y cómo decide el docente abordarlo en cada clase dependiendo del tema a desarrollar, pues el proceso de aprender se liga al de comprender para lo cual será perentoria la puesta en marcha de los procesos de memoria (Jiménez, 1998).

El docente puede considerarse entonces, el responsable de estimular en mayor o menor medida, los procesos de memoria como facilitadores del proceso de adquisición de conocimientos, para lo cual se requiere 
que en la misma formación de quienes educan se promueva la incorporación de estrategias didácticas que potencien las capacidades cognoscitivas básicas del alumnado. En este trabajo, se explora entonces, el conocimiento que posee el cuerpo docente sobre una de esas capacidades, a saber; los procesos de memoria, pues aproximarse a qué conoce sobre estos posibilitará pensar investigaciones futuras que indaguen las implicancias de la memoria en el aprendizaje.

\section{EsTADO DE LA CUESTIÓN}

Este estudio se centró en el conocimiento que poseen docentes sobre la memoria y cómo esto se ve reflejado en la planificación y el desarrollo de las clases. Asimismo, se indagó sobre los efectos de los distintos tipos de memoria en los procesos de aprendizaje, para así poder conjeturar sobre su implicancia en la calidad educativa del alumnado.

En este punto es necesario pensar sobre la calidad de la educación y los factores que en ella intervienen. Uno de ellos tiene que ver con la formación académica del profesorado y su posterior capacitación. En tal sentido, tanto la experiencia como la investigación han confirmado que uno de los factores claves para conseguir una educación de calidad es contar con docentes de calidad (Sandoval, 2009).

En la misma línea, Campos (2010) se pregunta ¿por qué frente a tantas iniciativas no se logra mejorar la calidad de los sistemas educativos? Y contempla la idea de que la calidad educativa se encuentra estrechamente ligada a la calidad del docente.

En sintonía, el informe Theachers and Educational Quality: Monitoring Global Needs for 2015 (UNESCO, 2006) destaca que no solo juega un papel fundamental la formación inicial del docente, sino que resalta la importancia y suma necesidad de que este siga formándose a lo largo de su trayectoria profesional.

Al hablar de medios apropiados para una innovación o transformación de la educación y de la práctica pedagógica, corresponde en primer lugar entender qué será transformado. El ser humano está dotado no solamente de habilidades cognitivas, de razón, sino también de habilidades emocionales, sociales, morales, físicas y espirituales, todas ellas provenientes del más noble órgano de su cuerpo: el cerebro. En el cerebro se encuentra la respuesta para la trasformación y es en él donde ocurrirá la transformación: en el cerebro del docente y en el cerebro del alumnado (Campos, 2010, p.3).

Siguiendo con esta investigación se determina que la habilidad de adquirir, formar, conservar y recordar la información depende de factores endógenos y exógenos, de las experiencias y de la metodología de aprendizaje. Es por eso que, para la/el docente, es de suma importancia conocer cómo aprende el cerebro, es decir cómo aprende, procesa, registra, conserva y evoca una información, para así poder mejor sus propuestas áulicas; por ende, todos los/las educadores/as deben conocer los procesos de memoria y cómo actuar sobre cada uno de ellos (Campos, 2010).

Continuando con esta línea de pensamiento, se puede aludir a una investigación sobre la importancia de la memoria en la enseñanza de inglés como lengua extranjera. En esta investigación, la autora elabora un repaso por la historia de la didáctica de inglés y afirma que la mejor manera que una persona tiene de aprender una lengua extranjera es utilizando la memoria, y los principios de la memoria, a los cuales se define en el artículo como atención, objeto de aprendizaje, motivación, asociación y repetición. A su vez, remarca que es imprescindible, contextualizar cada tema por desarrollar con la realidad del sujeto que aprende. Y remarca que aunque un aprendizaje significativo no debe basarse únicamente en la memoria, esta es absolutamente imprescindible para aquel (Jiménez, 1997).

En este sentido Morgado (2005) resalta que "aprendizaje y memoria son procesos cerebrales estrechamente ligados que originan cambios adaptativos en la conducta. La estabilización de los cambios neurales que tienen lugar tras el aprendizaje permite la consolidación de las memorias y su mantenimiento a largo plazo" (p. 295). El aprendizaje y la memoria son las funciones que permiten la adaptación al medio y la construcción de la propia historia como seres únicos. 
Retomando la idea de la necesidad de que el docente conozca y comprenda los procesos cognitivos, Flores (2016) sostiene que es importante tener en cuenta cómo funciona la inteligencia infantil para actuar en la dimensión cognitiva, dimensión importante para la adquisición de conocimientos y el aprendizaje. Al mismo tiempo que remarca la importancia de estimular la memoria operativa o memoria de trabajo específicamente para mejorar los procesos de aprendizaje.

Siguiendo esta línea, Etchepareborda y Abad (2005) resaltan la importancia de la memoria de trabajo en los procesos de aprendizaje, tal es su relevancia en dichos procesos que una afectación en los mecanismos básicos de este tipo de memoria provocaría una afectación en diversos procesos de aprendizaje formal académico, dificultando la inhibición de estímulos irrelevantes, el reconocimiento de patrones de prioridad, impedimento en formular una intención, dificultad en reconocer y seleccionar las metas adecuadas para la resolución de un problema, y un sinnúmero de procesos que se requiere para un aprendizaje significativo y de calidad.

Actualmente se observa que en educación el concepto de memoria ha sido tergiversado y convertido en un aspecto negativo del aprendizaje, por lo tanto, es necesario reivindicar la memoria en el ámbito educativo para así poder generar estrategias de aprendizaje que permitan el desarrollo de esta en el alumnado.

Es necesario circunscribir el término a un modelo que permita el incremento y desarrollo de otros niveles mentales, en los cuales las habilidades y características del maestro y el trabajo que desarrolla de forma intra áulica serán también un referente de determinante en esta nueva concepción (Hernández, Farfán, García, 2015, p. 7).

\section{MARCo TEÓRICO}

\section{Memoria y procesos de memoria}

Diversos autores se encargaron de conceptualizar y caracterizar la memoria, Luria (1984) postuló que se trata de la impresión, retención y reproducción de las huellas de la experiencia anterior que le permite al hombre acumular información. Desde este punto, la memoria es una red de sistemas interactivos, cada uno capaz de registrar y almacenar información y de hacerla disponible para su recuperación. Por su parte, Redolar (2012) define la memoria como una particularidad del funcionamiento cerebral, que se expresa mediante sistemas factibles de discriminarse por su estructura biológica y su funcionamiento.

Aristóteles, por ejemplo, creía que los recuerdos eran espíritus que viajaban por la sangre hasta el corazón. Hoy es posible comprobar en un escáner electrónico que, en realidad, son conexiones neuronales (Kundera, 2010). Los recuerdos están representados en el cerebro de alguna forma, pero la naturaleza de la huella que dejan, se llama engrama.

Vásquez (2015) plantea que la memoria no puede concebirse como un constructo unitario, pues son diversos los procesos específicos que son partícipes y favorecedores de la capacidad de memoria, lo cual posibilita la distinción de varios sistemas de memoria. "El concepto de sistemas de memoria hace referencia a los tipos de memoria que existen y que van desde fenómenos difícilmente conscientes para el sujeto hasta la memoria de nuestra vida" (Vásquez, 2015, p.118).

Los estímulos percibidos por los sentidos son procesados primeramente por el sistema de memoria denominado memoria sensorial, la cual presenta la particularidad de almacenar la información por un lapso muy breve (milisegundos) y se pone en funcionamiento sin el esfuerzo consciente, pues se activa automáticamente (Vásquez, 2015).

La memoria a corto plazo (MCP) es otro de los sistemas de memoria, siendo el encargado de almacenar información durante periodos cortos de tiempo, es una instancia de retención de la información de duración y capacidad limitada. Este tipo de memoria procesa de modo activo la información, y es también denominada memoria operativa (MO) o memoria de trabajo (MT) (Aguado, 2001). Si bien existen autores 
que sostienen que MCP, MO y MT son procesos semejantes, una postura interesante han adoptado Baddeley y Hitch (1974, citados en Vásquez, 2015) quienes plantearon la MT como un sistema cognitivo de mayor complejidad a la MCP, sosteniendo que esta última entendida como depósito de datos era insuficiente para explicar las manipulaciones y procesamientos que se desarrollan con la información por breves períodos, por lo que hoy puede afirmarse que la MCP se constituye en el almacén específico de la MT. (Vásquez, 2015)

Otro de los sistemas de memoria es la memoria a largo plazo (MLP), de mayor complejidad, en el que se encuentra almacenado todo lo que se ha aprendido y/o experimentado. Vásquez (2015) menciona que su nombre se debe a lo que la define, es decir, por la capacidad para almacenar información que permanece disponible para cuando sea requerida y en diferentes formatos por tiempos prolongados e incluso con una capacidad prácticamente ilimitada.

Dentro de la MLP se distinguen dos sistemas o funciones: memoria episódica (sucesos particulares de la historia del sujeto) y memoria semántica (conocimiento general de la vida de cada persona).

Además de poder distinguir diversos tipos de memoria, es factible reconocer que el proceso de memoria se produce en tres etapas básicas, a saber: codificación, almacenamiento y recuperación (Baddeley, 1997 en Piedra y Cartín, 2011).

La codificación implica captar la información mediante la conformación de una huella mnémica, la cual se asocia a datos previamente almacenados en la memoria. Cabe destacar que la información se almacena codificada, es decir, que no es factible conservar los datos como son percibidos, debido a que existe un procesamiento inicial de selección de aquellos que son pertinentes, luego de una interpretación que atribuye significado, y finalmente se integra a estructuras previamente existentes (Manzanero, 2008). En este proceso, se involucra el hipocampo, el cual alberga la representación de mapas cognitivos y el cerebelo, está involucrado en la codificación de recuerdos complejos, desempeña un papel en el aprendizaje motor y de recuerdos procedimentales.

Cuando la información ya fue codificada, puede ser almacenada en la memoria a corto plazo o a largo plazo. En esta fase de almacenamiento o conservación, se producen sucesivas recodificaciones de la información almacenada, este es el proceso más importante de la fase de almacenamiento; la recodificación, se define como los procesos y operaciones que tienen lugar después de la codificación de un suceso original y que provocan cambios en las huellas de memoria. La estructura neurobiológica involucrada en este proceso es la amígdala, interviene en el proceso de transferir los conocimientos desde la MCP o la MT a la MLP.

Finalmente, tiene lugar el proceso de recuperación, para el que se requiere una serie de requisitos, como ser: la predisposición del sistema cognitivo para evocar determinada información requerida, la presencia de estímulos o pistas que den iniciativa a la recuperación de información almacenada (Fierro, 2012).

\section{Aprendizaje}

No existe ninguna teoría que responda satisfactoriamente a la pregunta ¿Qué es aprender?, aun así, hay diversas teorías que se ocupan de definir el aprendizaje. En este trabajo se considera que

El aprendizaje es el proceso de construcción y apropiación del conocimiento que se da por la interacción entre los saberes previos del sujeto y ciertas particularidades del objeto. Proceso que se dan en situación de interacción social con pares en el que el docente interviene como mediador del saber a enseñar (Filidoro, 2004, p.3).

Se ha mencionado que aprendizaje y memoria son procesos íntimamente relacionados, y como se ha probado en diferentes estudios; para poder aprender, el cerebro debe retener cierta información, y para poder memorizar, el cerebro precisa la incorporación de información, por lo que podría decirse, que memoria y aprendizaje son dos momentos de un mismo proceso (Aguado, 2001).

Espinoza, Puma, Carrión y Aguilar (2010) asocian los procesos de aprendizaje y memoria con la neuroplasticidad, estableciendo que aprender necesariamente involucra modificaciones en diferentes niveles, 
a saber: cognitivo, conductual y anatómicos-fisiológicos; en este sentido el conocimiento adquirido y almacenado tiene asiento en redes neuronales diversas; al mismo tiempo que aprender y memorizar favorecen la neuroplasticidad.

El aprendizaje y la memoria no son procesos puntuales que ocurren en un sitio cerebral determinado, sino que, son estados funcionales para los que se requiere la participación de numerosas estructuras nerviosas y la correcta activación temporal entre ellas. Por ello, el aprendizaje depende del estado motivacional y emocional del individuo que aprende, del grado de atención, de sus conocimientos y habilidades previas, de sus receptores sensoriales, así como del estado de sus músculos, en el caso que se trate de aprendizaje motor (Espinoza et ál. 2010, p. 3).

\section{Procedimiento metodológico:}

Diseño de investigación. Se optó por una investigación cuantitativa, específicamente de diseño preexperimental, de preprueba - posprueba con un solo grupo con un grado mínimo de control; con el fin de efectuar un primer acercamiento del tema planteado.

Alcance. Este estudio tiene alcance exploratorio, para así familiarizarse con un tema poco abordado, visualizar relaciones potenciales entre las variables memoria-aprendizaje y obtener datos para el desarrollo de una posterior investigación de mayor rigurosidad y profundidad.

Acercamiento al campo. El trabajo de campo se ejecutó en la ciudad de Resistencia, Chaco, República Argentina, en dos momentos; preprueba (antes de la capacitación) y posprueba (al finalizar las jornadas de capacitación).

Fuentes. La información fue recolectada mediante encuestas confeccionadas para el estudio, respondidas por las y los docentes, por lo que la fuente se constituye como primaria.

Participantes. La muestra fue no probabilística por conveniencia, se seleccionó de manera no arbitraria, participaron un total de 196 personas, el $8 \%$ de sexo masculino y el $91 \%$ de sexo femenino. Un alto porcentaje de participantes pertenecían al ámbito de la educación, ya sea nivel inicial, primario, secundario, educación especial o estudiantes de dichas modalidades.

\section{Caracterización de la muestra}

En el Gráfico 1, se observa la conformación de la muestra en relación con el género de los participantes; el $91 \%$ eran mujeres, y el $8 \%$ hombres.

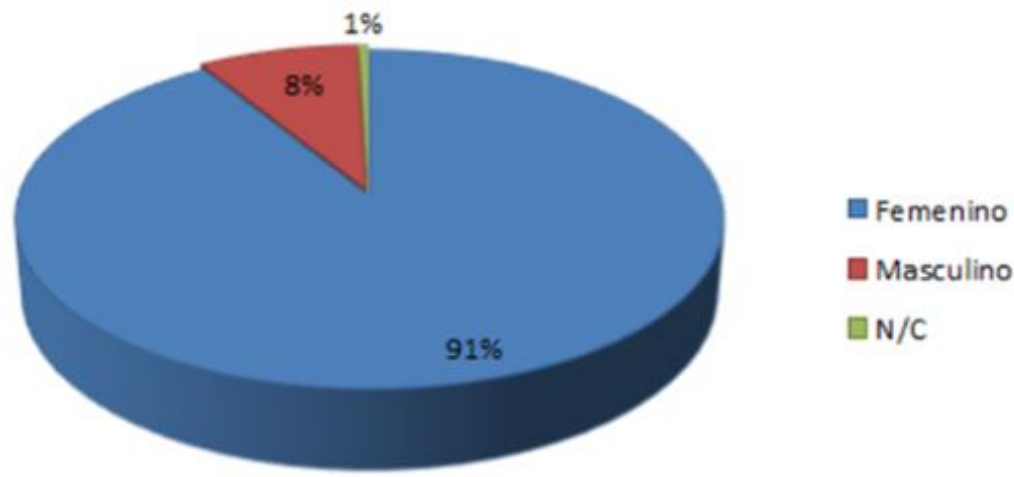

GRÁFICO 1

Género de los participantes

Fuente: Elaboración propia 
En el Gráfico 2, se distribuye la muestra en función del grado de formación de los participantes. El 85\% de los participantes ya había finalizado sus estudios de grado al momento de la Jornada, el 14\% aún se encontraba estudiando.

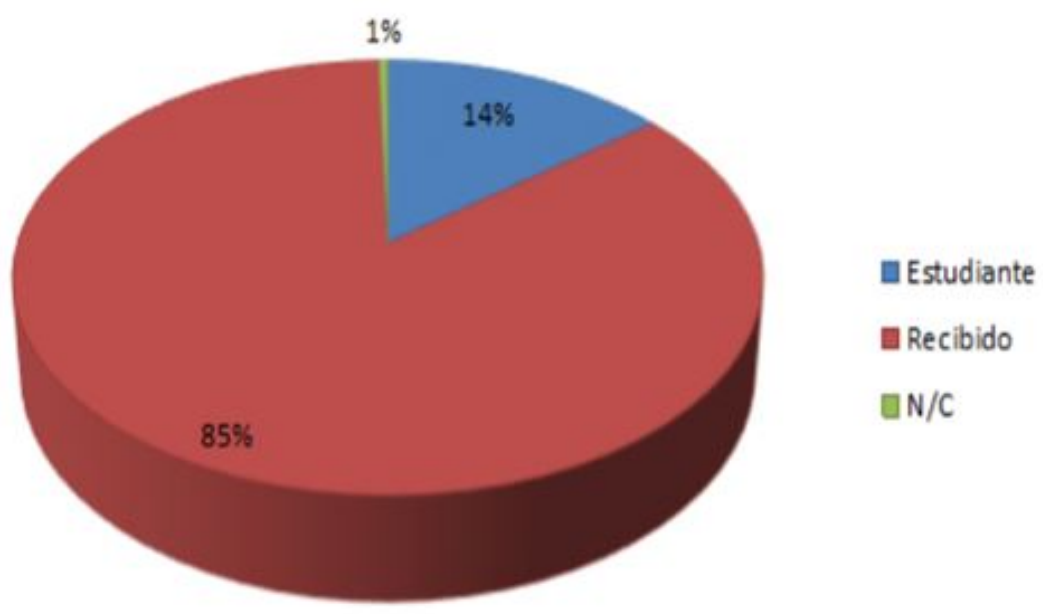

GRÁFICO 2

Grado de formación de los asistentes a las jornadas

Fuente: Elaboración propia

En el Gráfico 3, se detalla el porcentaje de los participantes en relación con el nivel en donde se desenvuelven profesionalmente; el $29 \%$ de la muestra se encuentra en nivel especial, el $26 \%$ en el nivel secundario, $17 \%$ en nivel primario, el $12 \%$ en nivel inicial, el $11 \%$ en otros niveles no especificados, y el $5 \%$ no se conoce.

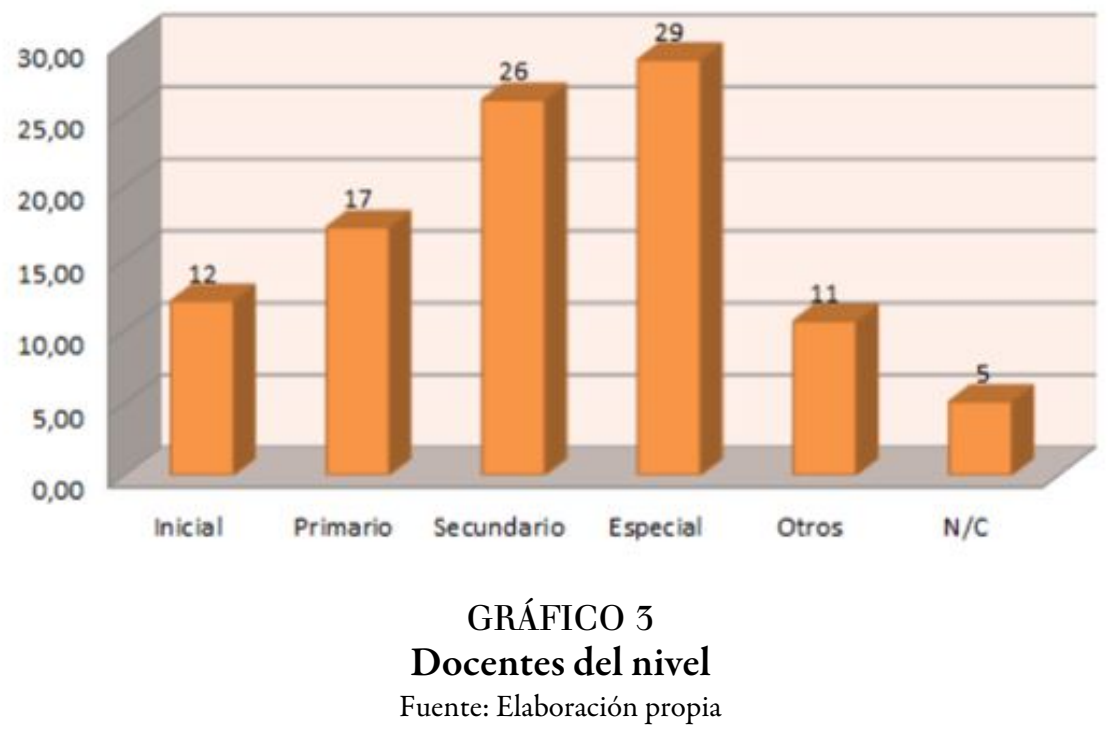

En el Gráfico 4, se expone la distribución de los participantes en relación con la antigüedad en la docencia; el 46\% tiene entre 0 y 5 años de antigüedad, el 16\% entre 6 y 10 años, el $10 \%$ entre 11 y 15 años, el $7 \%$ entre los 16 y 20 años, y el 7\% superaron los 21 años de antigüedad en la docencia y no se conoce la antigüedad del $14 \%$ de la muestra. 


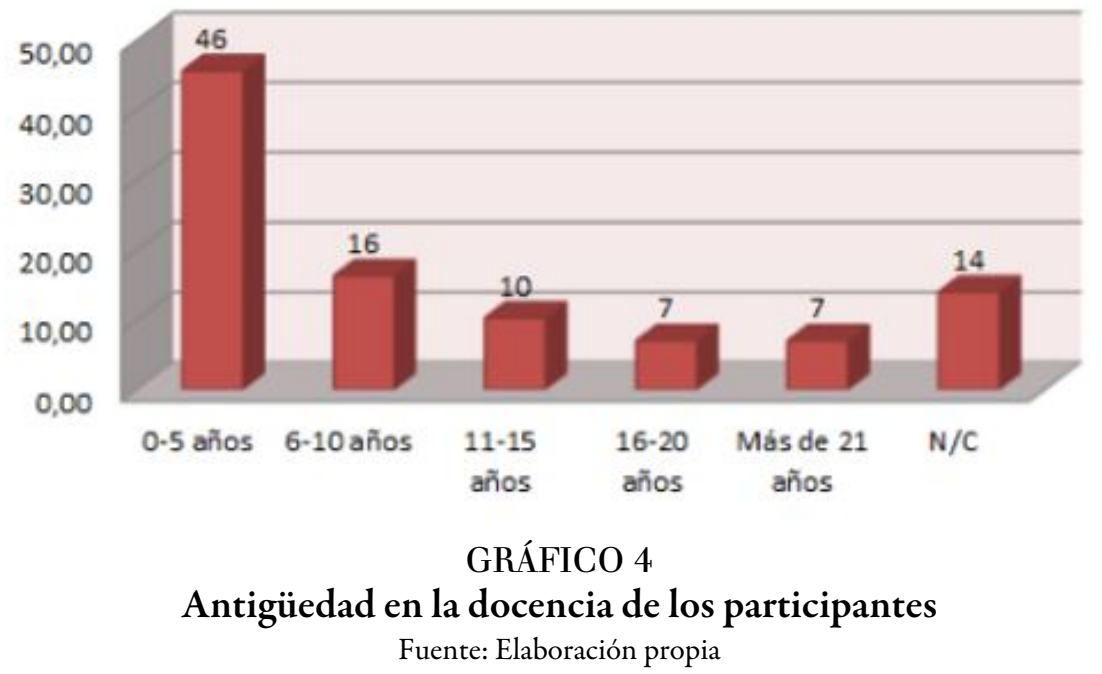

En el Gráfico 5, se caracteriza a la muestra en función de su formación en neurociencias; se denota que el $48 \%$ nunca habían recibido capacitación en neurociencias, el 19\% efectuó cursos relacionados a las neurociencias, el 14\% recibió formación en neurociencias como parte del profesorado, el 3\% había desarrollado posgrados en neurociencia, no se conoce la respuesta del 16\% de la muestra.

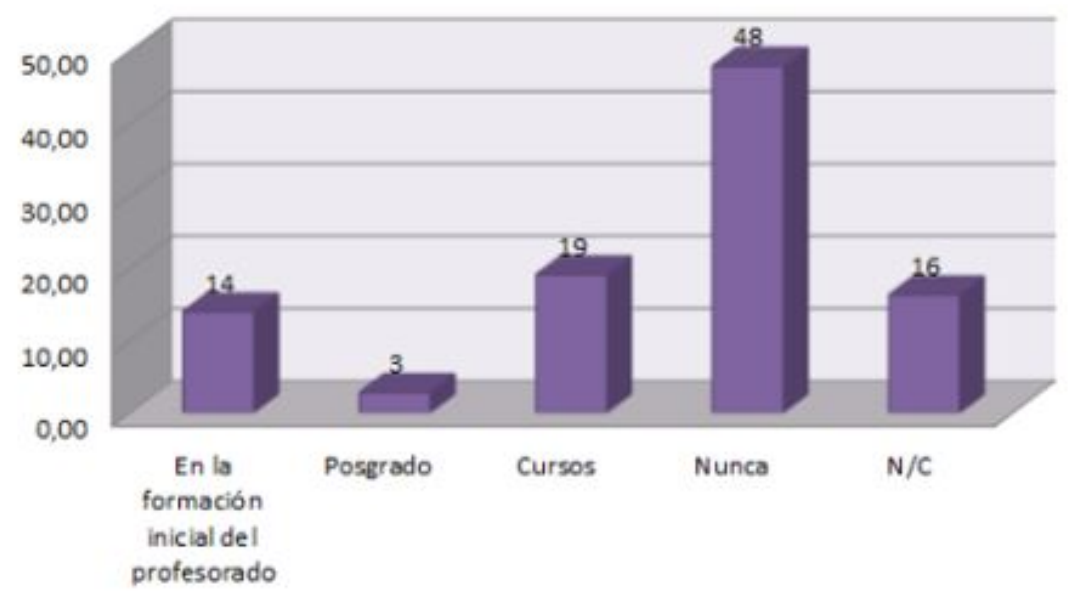

GRÁFICO 5

Formación en neurociencias de los participantes

Fuente: Elaboración propia

Procedimiento. En el marco de una jornada de capacitación en neuroeducación, en conformidad con el diseño de investigación propuesto, se inicia la etapa preexperimental de este estudio con la entrega de una encuesta para indagar el conocimiento de las y los docentes sobre los procesos de memoria. De este modo, previo al inicio de la capacitación, se les solicitó que completen la primera parte de la encuesta. Seguidamente se dio curso a la capacitación; para finalmente en la etapa posprueba solicitar completar la segunda parte de la encuesta.

Herramienta de recolección. Se ideó y confeccionó una encuesta ad hoc, específica para la jornada, de carácter anónimo. Dicho instrumento denominado Encuesta a Docentes sobre los Pasos de la Memoria consta de un espacio donde el docente completa: género, grado de formación, nivel en el que se desempeña como docente, antigüedad en la docencia, otro tipo de formación profesional (para no docentes), si posee o no formación en 
neurociencias. A continuación, la encuesta expone una primera parte titulada ¿Qué sabemos de los procesos de memoria? en la cual se presentan seis preguntas que indagan el conocimiento previo del cuerpo docente en relación con la memoria, sus tipos y su rol en el aprendizaje desde su función educador. Una segunda parte titulada ¿Qué aprendimos sobre la memoria? Busca sondear, mediante cuatro preguntas cerradas de opción múltiple, la información que han obtenido/retenido los asistentes a la jornada de capacitación respecto a las mismas variables indagadas en la primera etapa, incorporando además dos preguntas; la primera explora los procesos de memoria en los cuales cada docente enfatiza en su práctica pedagógica, y la segunda que lo invita a mencionar cuáles son los procesos de memoria que debería incorporar en su práctica.

Técnica de análisis y sistematización de resultados. Los datos obtenidos se procesaron mediante el empleo de hojas de cálculo Excel obteniendo porcentajes en las diversas variables observadas. La primera parte de la encuesta requirió un análisis previo a la carga de los datos para su procesamiento; pues se agruparon las respuestas a las preguntas abiertas en tres categorías: conoce, conoce poco y desconoce, con el objetivo de cuantificar los datos cualitativos y ejecutar las interpretaciones con base en porcentajes. En relación con la segunda parte de la encuesta, los datos a las preguntas de opción múltiple se cargaron según las opciones (A, $\mathrm{B}$ o C), siendo una de ellas la acertada, mientras que las respuestas a las dos preguntas abiertas planteadas se procesaron según su frecuencia de aparición en la muestra participante.

\section{ANÁLISIS Y DISCUSIÓN DE LOS RESULTADOS}

Se ha procurado efectuar una aproximación al campo de conocimiento que posee el docente respecto a los procesos de memoria. Mediante la encuesta elaborada a los fines de este estudio se obtuvieron datos que sientan las bases para líneas de investigación por emprender.

Conforme a los objetivos planteados en este estudio, se exponen a continuación los resultados a los que se arribaron. Específicamente al respecto de cuáles son los conocimientos que posee el personal docente sobre la memoria y sus etapas, en el Gráfico 6 puede observarse que en un primer momento (preprueba) gran parte de los asistentes (65\%) respondió que conoce poco; solo el 18\% manifestó conocer qué es la memoria, y un $15 \%$ expresó desconocer este proceso cognitivo.
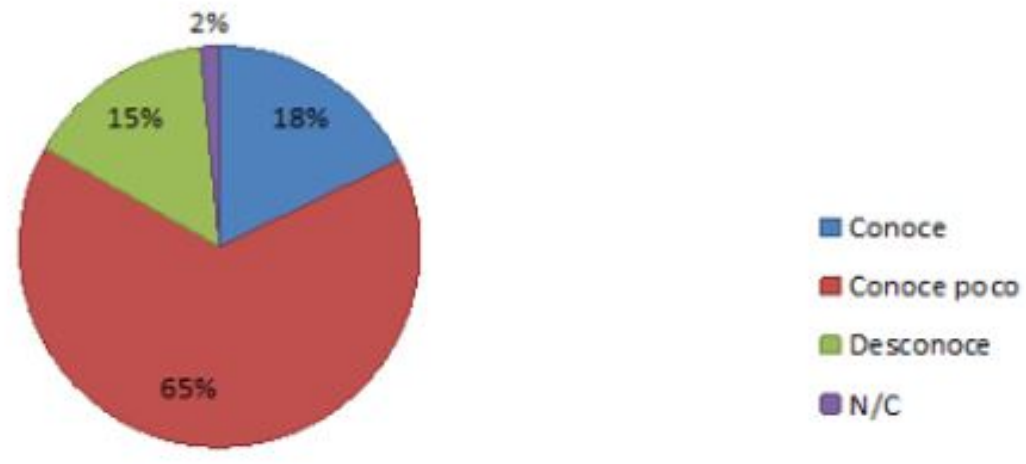

$$
\begin{gathered}
\text { GRÁFICO } 6 \\
\text { ¿Qué es la memoria? } \\
\text { Fuente: Elaboración propia }
\end{gathered}
$$

Por otra parte, en el Gráfico 7, las respuestas se clasificaron en función de los tipos de memoria que reconocían los participantes, observándose que el $44 \%$ reconoce todos los tipos de memoria, el $37 \%$ conoce corto y largo plazo, el $14 \%$ conoce únicamente memoria sensorial. 


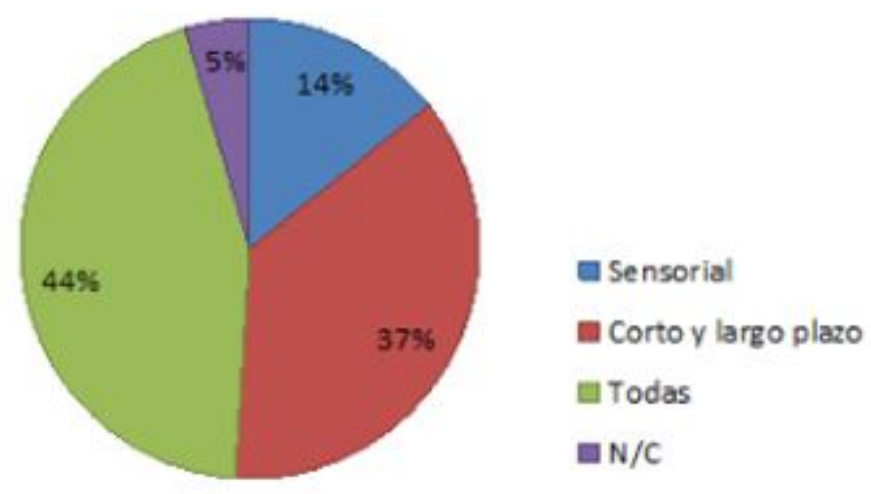

\section{GRÁFICO 7 \\ Tipos de memoria}

Fuente: Elaboración propia

Además, fue posible apreciar que al interrogar sobre la relación entre las variables memoria y aprendizaje, una amplia mayoría de los asistentes (72\%) expusieron conocer poco de esta, un $13 \%$ plantearon conocerla y un $13 \%$ desconoce tal vinculación; tal y como puede apreciarse en el Gráfico 8.
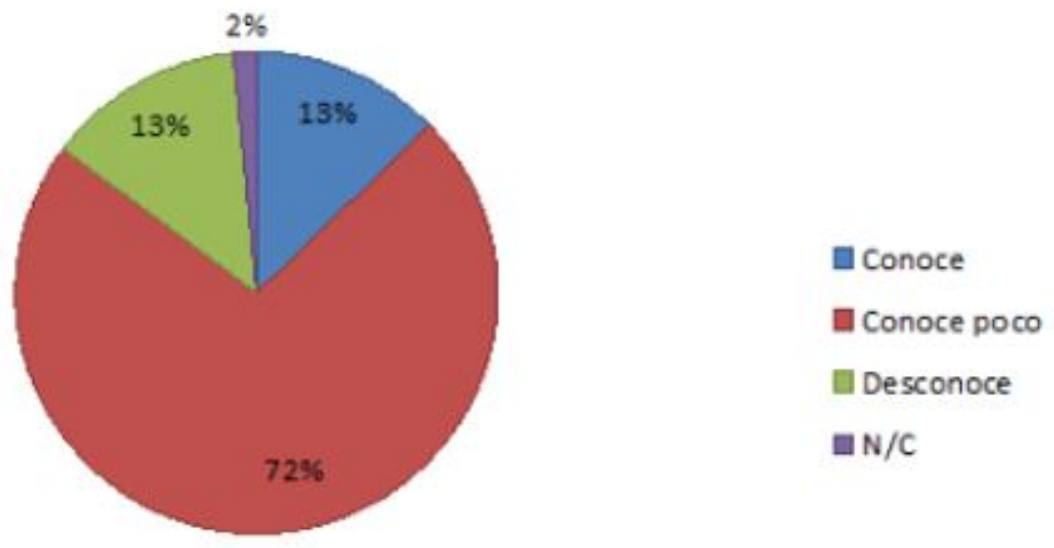

\section{GRÁFICO 8 \\ Relación memoria-aprendizaje \\ Fuente: Elaboración propia}

Luego de los talleres ejecutados en las Jornadas de Neuroeducación, se les pidió a los participantes que completaran la segunda parte de la encuesta, la cual se relacionaba con los temas tratados a lo largo de los días de capacitación. Al analizar los datos, los resultados fueron los siguientes:

En el Gráfico 9, se observa la respuesta de los participantes en relación con ¿Qué es la memoria?; la gran mayoría de los asistentes (95\%) ha concebido a la memoria como un proceso neurocognitivo que permite registrar, codificar, consolidar, almacenar, acceder y recuperar la información (opción B). El 3\% definió a la memoria como un proceso mental complejo que posibilita al ser humano atender a los estímulos relevantes de entre otra información presente en el medio ambiente (opción C). Y un 2\% no respondió la consigna. 

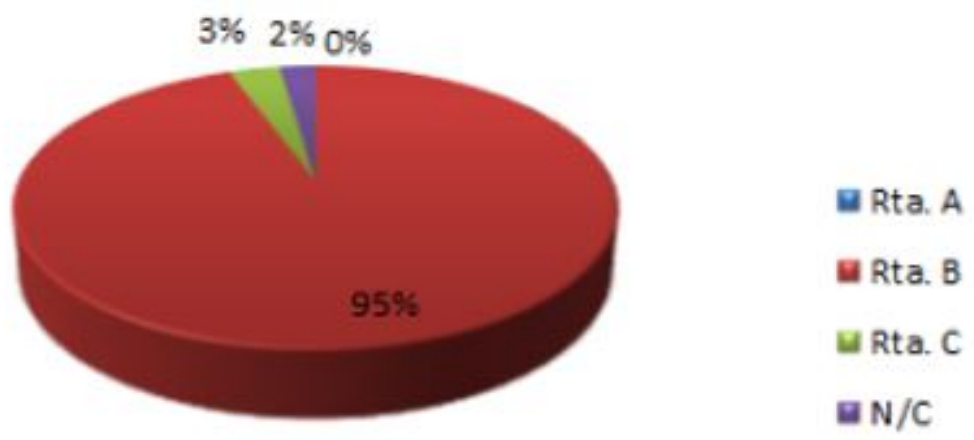

GRÁFICO 9

Respuesta de los participantes sobre ¿qué es la memoria?

Fuente: Elaboración propia

Similares resultados se obtuvieron al explorar los tipos de memoria que el profesorado conocía, luego de las jornadas de capacitación. En el Gráfico 10, puede apreciarse que el 96\% expresó conocer todas las memorias, el $1 \%$ conocía la memoria a corto y largo plazo, el $1 \%$ contesto conocer solo memoria sensorial, el $0 \%$ conocía memoria sensorial y a corto plazo, y el $0 \%$ no respondió a la consigna.

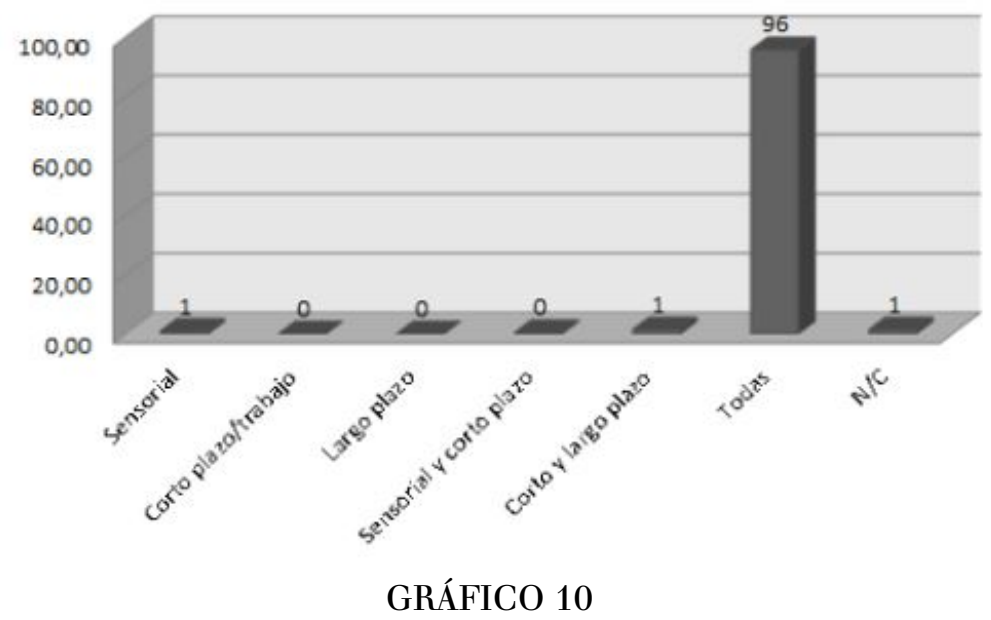

Respuesta de participantes sobre ¿qué tipos de memoria conocen?

Fuente: Elaboración propia

En relación con lo anterior, en el Gráfico 11, se ilustran las respuestas de los participantes a la pregunta ¿Cuáles son los procesos de memoria? El 91\% considera que los procesos de memoria son codificación, almacenamiento y recuperación, respondiendo correctamente (opción $\mathrm{C}$ ), el $8 \%$ reconoce como procesos de la memoria a la percepción, codificación y recuperación (opción B) y un mínimo porcentaje no responde (1\%). 


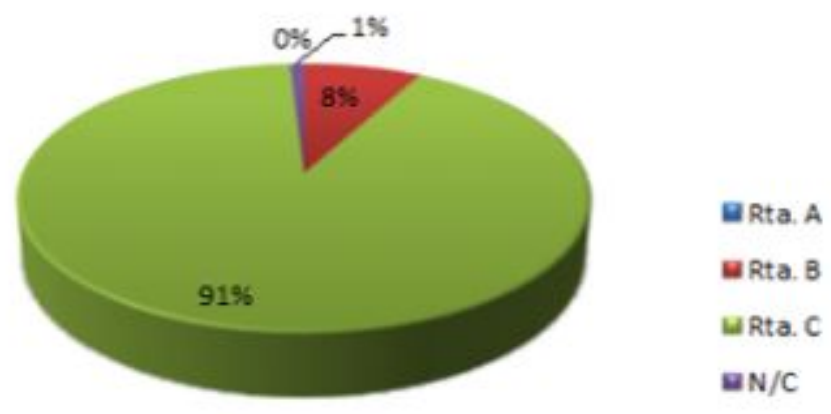

GRÁFICO 11

¿Cuáles son los procesos de la memoria?

Fuente: Elaboración propia

En el Gráfico 12, pueden observarse los resultados en relación con la pregunta ¿Cómo se relaciona la memoria y el aprendizaje?, a partir de lo que pudo conocerse la relevancia que el grupo docente dice asignar a la memora en relación con el aprendizaje. De este modo, el $91 \%$ considera que la memoria siempre conlleva adquisición de la información y todo aprendizaje implica conservación y recuperación de la información, además de considerar que el aprendizaje depende de la memoria para su permanencia e, inversamente, la memoria precisa del contenido al que da lugar el aprendizaje (opción C). El 8\% considera que el aprendizaje depende de la memoria para su permanencia e, inversamente, la memoria precisa del contenido al que da lugar el aprendizaje, el $1 \%$ de la muestra no responde a la pregunta.

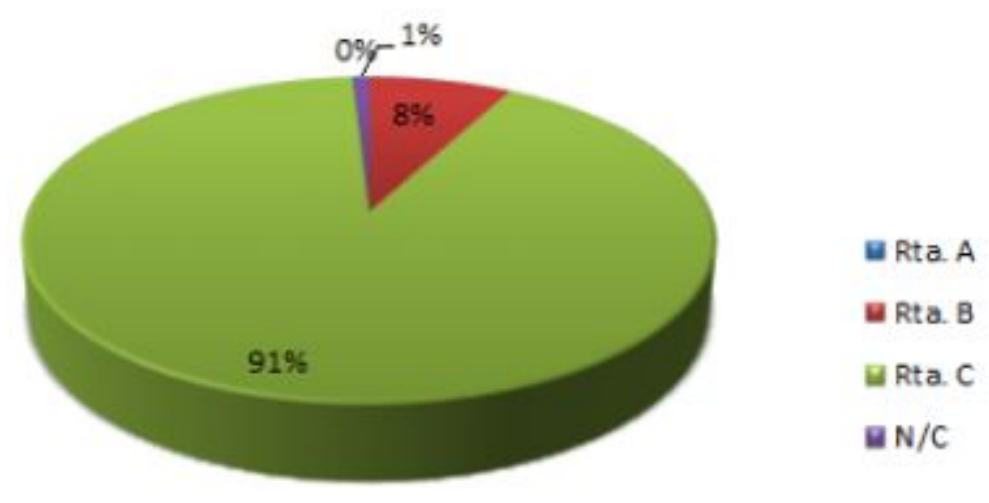

GRÁFICO 12

¿Cómo se relacionan memoria y aprendizaje?

Fuente: Elaboración propia

Teniendo presente los resultados hasta aquí obtenidos se puede inferir que la formación continua, en este caso mediante las jornadas sobre neuroeducación, surten efectos en los saberes de las y los docentes, pudiendo constituirse en contenidos presentes al momento de pensar y llevar adelante sus prácticas pedagógicas.

Ante la invitación (mediante pregunta abierta, en la segunda etapa del cuestionario) a pensar la propia práctica pedagógica en relación con la variable procesos de memoria, se ve en el Gráfico 13 que se pudo apreciar que un $26 \%$ de las y los docentes hace mayor hincapié en la codificación, el $24 \%$ expresa hacer énfasis en todos los procesos, el $9 \%$ se centra en la recuperación, el 6\% en el almacenamiento, el 5\% en la codificación y almacenamiento, el $4 \%$ hace énfasis en la codificación y recuperación, el $2 \%$ el almacenamiento y recuperación, y el $24 \%$ restante no respondió la consigna. Con base en estos resultados, se evidencia que un alto porcentaje de docentes se dedica en mayor medida a la adquisición de información por parte de los 
estudiantes, siendo probable que no se retome el tema dado, por ende, quedaría pendiente una exploración más exhaustiva al respecto de si en las clases se efectúan actividades que den lugar a la comprobación de almacenamiento de nuevos saberes y su posterior recuperación.
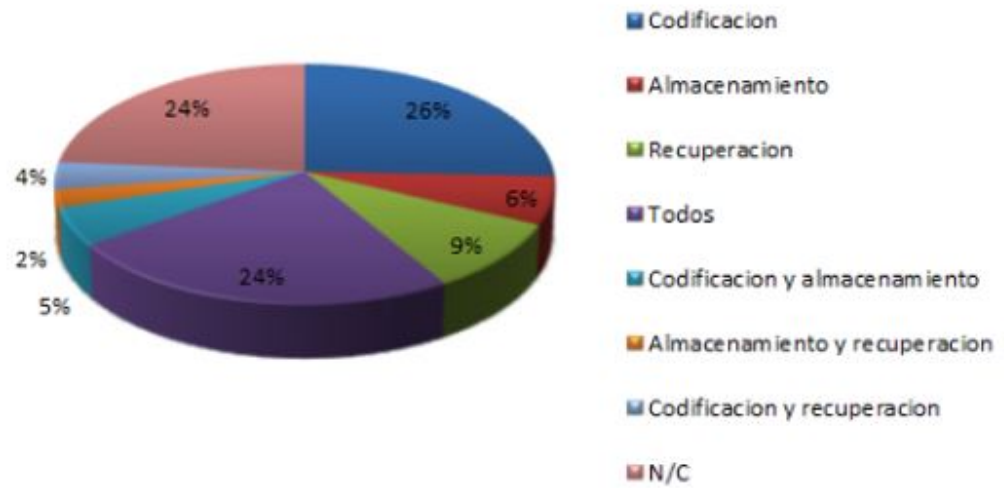

GRÁFICO 13

Respuesta de los participantes sobre: ¿En cuál de los procesos de memoria enfatizan sus prácticas pedagógicas?

Fuente: Elaboración propia

En el Gráfico 14, se puede apreciar la información respecto a ¿Cuál de los procesos de memoria debería incorporar en su práctica docente? El 29\% contestó que debería hacer más hincapié en todos los procesos, el $26 \%$ en codificación y almacenamiento, el $16 \%$ en recuperación, el $12 \%$ en codificación, el $10 \%$ en almacenamiento y el $4 \%$ en almacenamiento y recuperación.
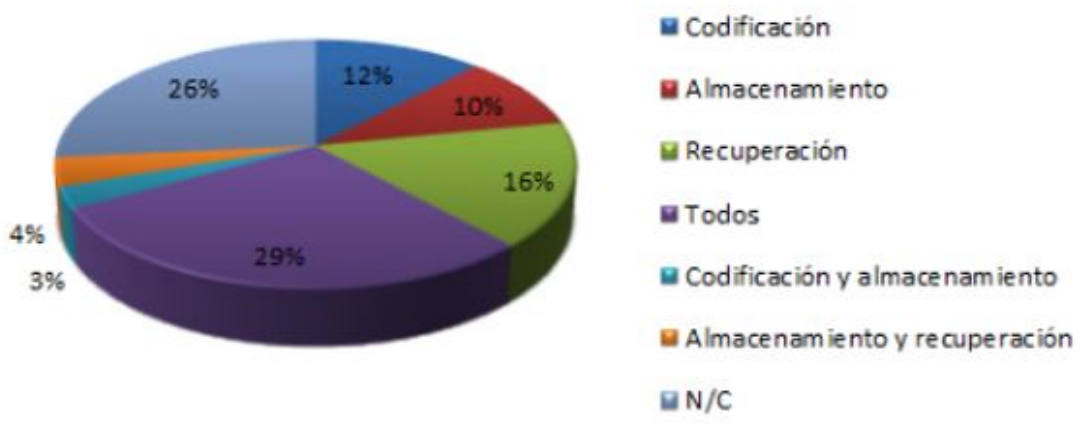

GRÁFICO 14

¿Cuál/es de los procesos de memoria debería incorporar en su práctica docente?

Fuente: Elaboración propia

En el Gráfico 15, se describe el incremento de las respuestas correctas en la encuesta antes y después de la jornada; estas aumentaron un $76 \%$ 


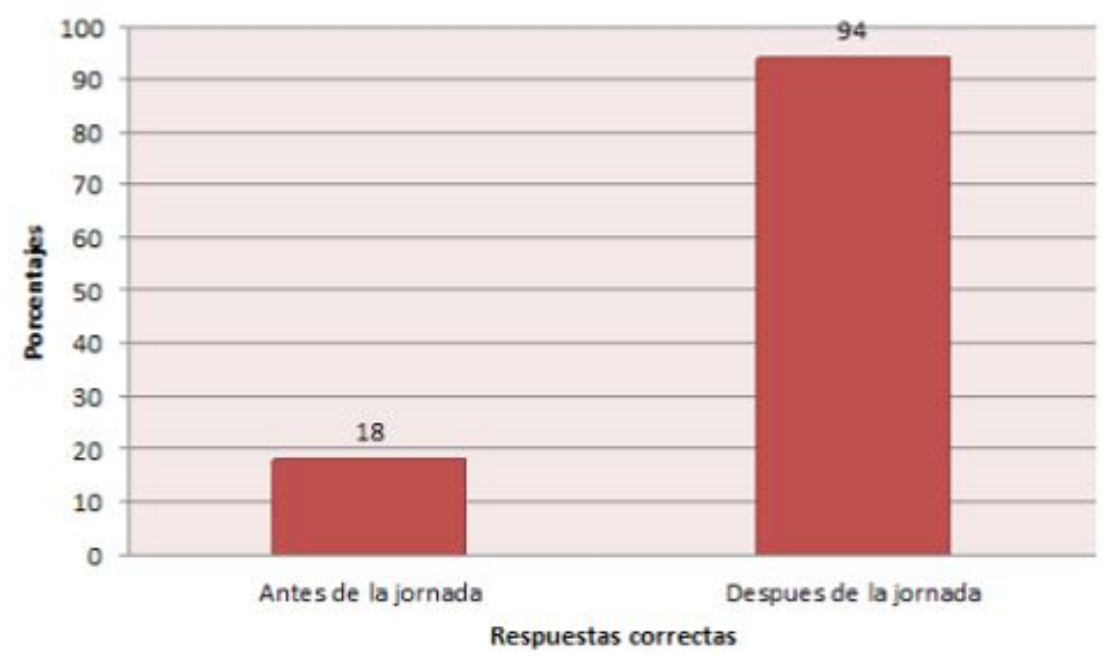

GRÁFICO 15

¿Qué es la memoria?

Fuente: Elaboración propia

En el Gráfico 16, se observa un incremento del 52\% en relación con el conocimiento de todos los tipos de memoria. Asimismo, disminuyó significativamente las respuestas que afirmaban conocer únicamente la memoria sensorial y de corto y largo plazo, en un $13 \%$ y $35 \%$ respectivamente.

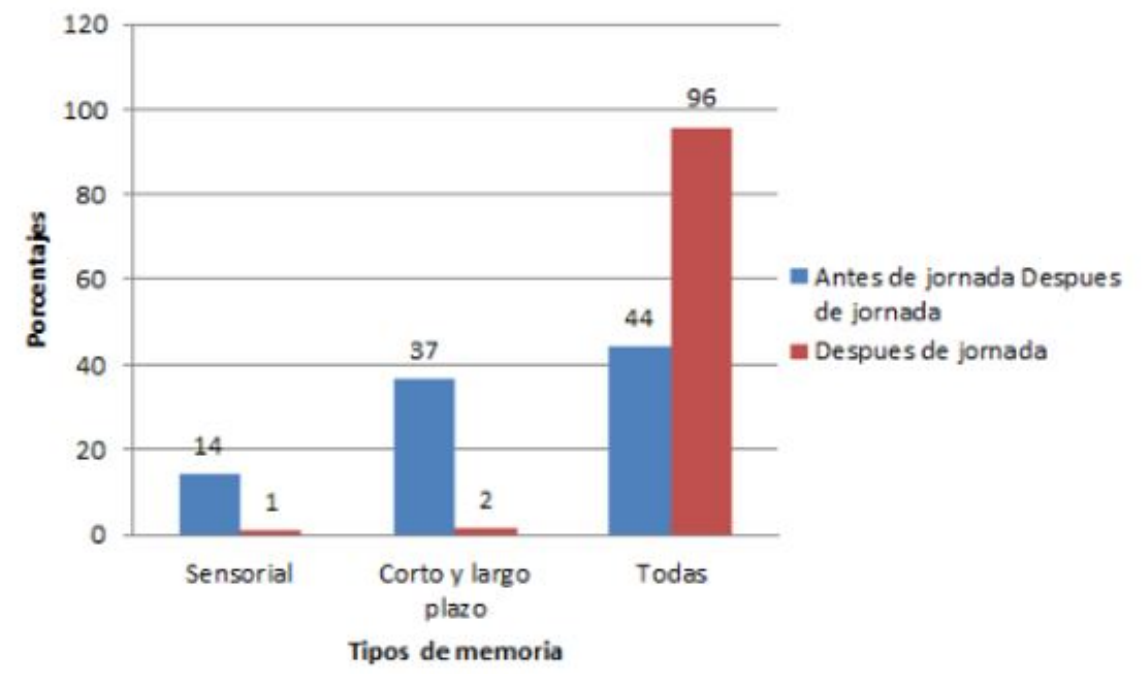

GRÁFICO 16

Tipos de memoria

Fuente: Elaboración propia

En el Gráfico 17, se destaca el aumento en el conocimiento de los asistentes respecto a la relación entre memoria y aprendizaje. Su incremento fue del $55 \%$ teniendo en cuenta la primera encuesta. 


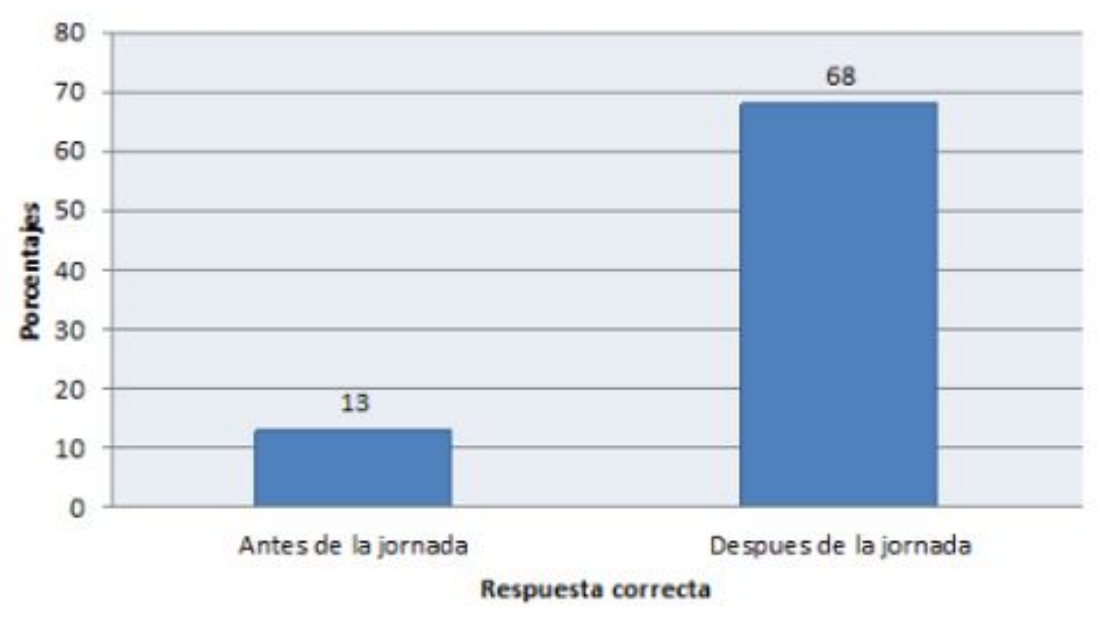

GRÁFICO 17

Relación Memoria-Aprendizaje.

Fuente: Elaboración propia

Se considera que es menester el estudio de asignaturas vinculadas al funcionamiento del cerebro y los procesos cognitivos involucrados necesariamente en el proceso de aprendizaje de los estudiantes, para lograr saberes significativos y efectivos. Dicha afirmación tiene sus bases en los resultados observados en el estudio de datos obtenidos de las encuestas en las Jornadas de Neuroeducación.

En este sentido, para que exista una educación de calidad, el docente debe tener conocimientos acerca del cerebro y su funcionamiento. Conocer sus habilidades y capacidades permitirá a cada docente poder planificar sus clases para mejorar y asegurar el aprendizaje en cada alumno (Campos, 2010). Asimismo, es imprescindible que cada docente conozca sobre los tipos de memoria y los procesos de esta, pues permitirá acercar actividades a las y los alumnos para mejorar el rendimiento y garantizar la adquisición de nueva información. Sin embargo, en la actualidad, se advierte que en los planes de estudio de formación docente no se cuentan con contenidos que den el protagonismo que se merecen los procesos de memoria como proceso cognitivo básico no solo para el aprendizaje sino también para la vida cotidiana del alumnado.

El conocimiento acerca de la forma que aprende el cerebro del estudiante, en cada etapa de su vida (niño, adolescente, adulto), brindará al docente la posibilidad de crear estrategias para abordar de la manera más adecuada la enseñanza de nuevos saberes, incluyendo en cada práctica educativa tanto los procesos de la memoria, como cada una de las habilidades cerebrales que el sujeto posee para aprender la una manera significativa.

Centrados específicamente a la memoria de trabajo (MT) Etchepareborda y Abad (2005) plantean que esta se conecta constantemente con la memoria a largo plazo, lo cual permite el acceso a los conocimientos y aprendizajes fijados anteriormente que se relacionan con el conocimiento que se mantiene online en la MT. Siguiendo la línea de este tipo de memoria, plantean que de acuerdo a investigaciones realizadas por diferentes autores, se encuentra una relación significativa entre los trastornos de aprendizaje, ya sea de aritmética o de lectura con dificultades en la memoria de trabajo (Etchepareborda y Abad, 2005). Al poseer conocimientos acerca de cómo funciona la memoria, las y los docentes podrán acceder a mayores y mejores estrategias de enseñanza, como por ejemplo saber que es necesario retomar un aprendizaje un tiempo después de haberlo aprendido, evocando los conocimientos para así poder fijar dicho conocimiento.

Otro conocimiento sumamente necesario y útil para las y los docentes es que al memorizar material con sentido y significado para las y los alumnos se requerirá menor tiempo y se obtendrá resultados más efectivos que si se memoriza material sin sentido; por ejemplo, con el aprendizaje de las tablas de multiplicar, se debe otorgarle un significado para que el alumnado pueda incorporarlas de mejor manera. (Téllez, 2003) 


\section{Conclusión}

A partir del abordaje de la problemática que motivó a desarrollar la investigación, se ha evidenciado que un gran porcentaje de docentes no contaba con conocimientos sobre la memoria, sus tipos y procesos, desconociendo la relación existente entre la memoria y el aprendizaje, sumado al hecho de que solo un bajo porcentaje reportó haber recibido formación en neurociencias en los profesorados, desconociendo los aportes que estas ofrecen al proceso de enseñanza y de aprendizaje.

Sobre las preguntas iniciales que circulan en las escuelas acerca de los olvidos del alumnado, se pudo observar que el cuerpo docente reportó hacer hincapié en los procesos de codificación siendo un bajo porcentaje el que involucra en su práctica pedagógica también los procesos de almacenamiento y recuperación, al menos de modo explícito/declarativo. Procesos tan necesarios como el primero, por lo cual los conocimientos trabajados con el alumnado al no ejecutar los pasos de la memoria necesarios (codificación, almacenamiento y recuperación) difícilmente puedan fijarse para poder ser utilizados posteriormente.

Se remarca la necesidad de contar con capacitaciones en neurociencias en relación con los procesos implicados en el aprendizaje, dado que después de la jornada sus respuestas mejoraron notablemente y pudieron comprender la interrelación existente entre memoria y aprendizaje.

Se considera que el conocimiento y la puesta en práctica de la memoria y sus procesos dentro de la planificación y práctica áulica, mejorarán notablemente el aprendizaje del alumnado en el nivel que se encuentren.

Por lo antes mencionado, se apunta a la necesidad de incorporar a los programas de formación docente el enfoque neurocientífico y el estudio del cerebro en sus distintas etapas evolutivas y la relación de los procesos cerebrales en el aprendizaje escolar. Asimismo, en indispensable la constante actualización y perfeccionamiento del personal docente de cada institución educativa, para mejorar día a día sus prácticas y fomentar el aprendizaje adecuado y efectivo en cada sujeto que aprende.

\section{Limitaciones}

Teniendo en cuenta que es un estudio cuyo alcance es exclusivamente exploratorio, las inferencias aquí efectuadas a partir del sondeo elaborado requieren de un análisis de mayor profundidad a partir del emprendimiento de investigaciones que indaguen la relación específica de los procesos de memoria con el aprendizaje; al mismo tiempo que se consideren los múltiples factores que intervienen en el proceso de enseñanza-aprendizaje.

\section{REFERENCIAS}

Aguado, L. (2001). Aprendizaje y memoria. Revista de neurología, 32 (4), 373-381.

Campos, A.L (2010). Neuroeducación: uniendo las neurociencias y la educación en la búsqueda del desarrollo humano. La educación revista digital (143), 1-14.

Davini, M. C. (2008) Métodos de enseñanza: didáctica general para maestros y profesores. Buenos Aires: Santillana.

Espinoza, G., Puma, E., Carrión, D. y Aguilar, L. (Agosto, 2010) Aprendizaje, memoria y neuroplasticidad. En A. L. Campos (Presidencia). I Congreso Mundial de Neuroeducación. Conferencia llevada a cabo en el congreso organizado por ASEDH-CEREBRUM, Lima, Perú.

Etchepareborda, M.C. y Abad, L. (2005). Memoria de trabajo en los procesos básicos del aprendizaje. Revista de neurología; 40 (1), 79-83.

Fierro, C. (2012) La memoria y emociones. Psicológica, Fisiológica y Conductualmente. Recuperado de https://bit.ly/ $2 \mathrm{YSrqdV}$ 
Filidoro, N. (2004) Psicopedagogia: conceptos y problemas. La especificidad de la intervención clínica. Buenos Aires, Argentina: Biblos.

Flores, M.C. (2016) El aprendizaje y la memoria de trabajo: un reto para el docente de hoy. (Tesis de maestría). Universidad de Manizales, Colombia.

Hernández, J., Farfán, J. y García, J. (2015). Reivindicando la memoria. Revista Iberoamericana de Producción Académica y Gestión Educativa, 2 (4), 1-9

Jiménez, R.M. (1997). La memoria y el aprendizaje de inglés en el Aula. Cauce: revista de filología y su didáctica, (20), 797-811.

Kundera, M. (2010). La memoria humana. Caracas: Banco Central de Venezuela.

Luria, A. (1984) El cerebro en acción. Barcelona: Ed. Martínez Roca.

Manzanero, A.L. (2008). Aspectos básicos de la memoria. En A. L. Manzanero (Ed.), Psicología del Testimonio (pp. 27-45). Madrid, España: Ed. Pirámide.

Morgado, I. (2005). Psicobiología del aprendizaje y la memoria: fundamentos y avances recientes. Revista de neurología; 40 (5), 289-297.

Piedra, L. y Cartín, J. (Febrero, 2011) La implicación de los procesos de memoria en la formación académica: una aproximación desde los resultados de una investigación sobre el estado de la cuestión en memoria. En M. D. Madrigal (Presidencia) II Congreso Internacional de Investigación Educativa. Conferencia llevada a cabo en el congreso organizado por el Instituto de Investigación en Educación, Universidad de Costa Rica, Costa Rica.

Redolar, D. (2012) Consolidación de la Memoria y Sustrato Nervioso del Refuerzo. Revista Argentina de Ciencias del Comportamiento, 4 (2), 51-74.

Sandoval, M. (2009.) Educación de Calidad y Desempeño Docente. Ánfora, 16 (27), 5-41.

Téllez, A. (2003) La memoria humana: revisión de los hallazgos recientes y propuestas de un modelo neuropsicológico. (Tesis de maestría). Universidad Autónoma de Nuevo León, Monterrey.

UNESCO (2006) Theachers and Educational Quality: Monitoring Global Needs for 2015. Recuperado de https://bi t.ly/2Me4JQ1

Vásquez, A. (2015) Manual de Introducción a la Psicología Cognitiva. Montevideo: UdelaR.

CC BY-NC-ND 